\title{
NEW VOLATILITY MODELS UNDER A BAYESIAN PERSPECTIVE: A CASE STUDY
}

\author{
Edilberto Cepeda Cuervo* \\ Jorge Alberto Achcar ${ }^{\dagger}$ \\ Milton Barossi-Filho $\ddagger$
}

\begin{abstract}
In this paper, we present a brief description of ARCH, GARCH and EGARCH models. Usually, their parameter estimates are obtained using maximum likelihood methods. Considering new methodological processes to model the volatilities of time series, we need to use other inference approach to get estimates for the parameters of the models, since we can encouter great difficulties in obtaining the maximum likelihood estimates due to the complexity of the likelihood function. In this way, we obtain the inferences for the volatilities of time series under a Bayesian approach, especially using popular simulation algorithms such as the Markov Chain Monte Carlo (MCMC) methods. As an application to illustrate the proposed methodology, we analyze a financial time series of the Gillette Company ranging from January, 1999 to May, 2003.
\end{abstract}

Keywords: ARCH; GARCH; EGARCH; Stochastic Volatility Models; Financial Time Series; Bayesian methodology; MCMC methods.

\section{Resumo}

Neste artigo, apresentamos uma breve descrição dos modelos ARCH, GARCH e EGARCH. Normalmente, as estimativas dos parâmetros desses modelos são obtidos através de métodos de máxima verossimilhança. Considerando-se novos processos metodológicos para modelar as volatilidades das séries temporais, precisamos usar outra abordagem de inferência para obter estimativas para os parâmetros dos modelos, uma vez que podemos ter grandes dificuldades para obter as estimativas de máxima verossimilhança, devido à complexidade da função de verossimilhança. Desta forma, obtemos as inferências para as volatilidades das séries temporais sob uma abordagem bayesiana, especialmente com o uso de algoritmos populares de simulação como o método de Monte Carlo em Cadeias de Markov (MCCM). Como uma aplicação para ilustrar a metodologia proposta, analisamos uma série temporal financeira da empresa Gillette variando de janeiro de 1999 à maio de 2003.

Palavras-chave: ARCH; GARCH; EGARCH; Modelos de Volatilidade Estocástica; Series de tempo Financieras; Métodos Bayesianos; Métodos de MCMC.

JEL classification: $\mathrm{C} 53$

DOI: http ://dx.doi.org/10.1590/1413-8050/ea91

\footnotetext{
*Universidad Nacional de Colombia. Departamento de Estatística. E-mail: ecepeda@unal.edu.co

† Universidade de São Paulo, FMRP, Brazil. Departamento de Medicina Social. E-mail: achcar@fmrp.usp.br

‡ Universidade de São Paulo, Brazil. Faculdade de Economia, Administração e Contabilidade de Ribeirão Preto. E-mail: mbarossi@usp.br
} 


\section{Introduction}

Financial time series volatility has been an issue that academics have focused on since Engle (1982) seminal paper. Mostly, volatility appeared to be an important issue after the abandonment of fixed parities dollar-gold by the USA at the beginning of the 70's. Since then, asset prices started changing more broadly, especially international exchange rates. After exchange raa' markets' volatility rising, it was just a matter of time for verifying the same in stock markets, mainly in the 80's and 90's. In order to frame this new phenomenon into feasible financial models, researchers kept on looking for alternative methodologies which reproduces excess volatility; a new stylized fact showed by financial time series.

Engle (1982) and Bollerslev (1986) offered a primary sort of answer to this issue, the so called ARCH class models. Intrinsically those models are leptokurtic and are, for instance able to capture the excess volatility contained in most financial time series. However this was not the first concern of Engle, whose applicative example take the UK inflation rates time series into account. Extending this empirical practice to other time series, mainly financial, was the next step.

After obtaining success on incorporating excess volatility into ARCH class models, another stylized fact arises from empirical data. Repeatedly, academics and financial analysts verified that financial volatility produced a skew impact on asset prices; i.e. bad news tends to impact more severely on asset prices than good news. In order to incorporate this stylized fact into volatility models, Nelson (1991) proposed an EGARCH class of models.

Though all these models recognized volatility is a latent variable, a more satisfactory answer to the problem of adjusting volatility as a stochastic model was provided by the stochastic volatility models. Hull \& White (1987) is a reference on this matter by adjusting a stochastic volatility process into option pricing formulas and, obtaining better results than the original Black \& Scholes (1973) option pricing model. In fact stochastic volatility models are a more flexible alternative for modeling financial time series, once one stochastic process is assumed for the random terms. However, these stochastic processes are still normal, guarantying a closed solution for the likelihood function.

From a methodological point of view Bayesian models are a generalization of those models briefly discussed above. Bayesian methods are more suitable for modeling financial time series and forecasting their future behavior as well, because they allow for an introduction of a broad variety of stochastic processes for describing latent variables; i.e. prior distributions. Definitely, classic inference dominates financial literature on modeling and forecasting time series behavior, however Bayesian inference is becoming a better alternative for the same reason, mainly after the rapid computational development in the 90s. Moreover, once Bayesian approach provides a broad way for modeling the stochastic term, better results can be obtained for estimating and forecasting financial time series behavior.

Another class of statistical models, the so-called stochastic volatility models (SV), has been a satisfactory alternative in analyzing financial time series, when compared to GARCH models. SV models are more flexible to model financial time series, given that they assume two processes for the noise. One process is for the observation and the other for the latent volatility. Compar- 
ative studies between SV models and models type GARCH are well known in the literature (see for example, Taylor 1994, Ghysels et al. 1996, Shephard 1996, Kim et al. 1998).

Bayesian Methods using Markov Chain Monte Carlo (MCMC) methods are considered in the analysis of financial time series assuming SV models (see for example, Meyer \& Yu 2000), given the great difficulties in the classical statistical approach with the complexity of the likelihood function.

This article is divided into 5 sections. In section 2 we define the ARCH in regression models proposed by Engle (1982), the generalized ARCH models or GARCH, introduced by Bollerslev (1986) and the exponential GARCH models or EGARCH, proposed by Nelson (1991). In section 3, we propose a Bayesian methodology to fit general exponential autoregressive models, GEGARCH. In section 4 , generalized stochastic volatility models or GSV, are defined. In section 5, we introduce a Bayesian analysis for GSV models. In section 6, the time series of prices and log-returns of the shares prices for the Gillette Company are analyzed, assuming different models for both time series. Finally, in section 7, we present some conclusions. The selection of the best model is made using the AIC (Akaike Information Criterion) and BIC (Bayes Information Criterion).

Finally, in section 7 , we present some conclusions.

\section{$2 \mathrm{ARCH}, \mathrm{GARCH}$ and EGARCH models}

\subsection{ARCH models}

If a random variable $y_{t}$ is drawn from the conditional density function $f\left(y_{t}\right)$ $\left.\psi_{t-1}\right)$, the forecast of today's value based on the past information $\psi_{t-1}$ is given by $\mu_{t}=E\left(y_{t} \mid \psi_{t-1}\right)$ and can be modeled as a regression model $\mu_{t}=x_{t}^{\prime} \beta$, where $x_{t}=\left(1, x_{t 1}, \ldots, x_{t q}\right)^{\prime}$ is the vector of the explanatory variables at time $t$, for example $x_{t}=\left(1, y_{t-1}, \ldots, y_{t-q}\right)^{\prime}$, and $\beta=\left(\beta_{0}, \beta_{1}, \ldots, \beta_{q}\right)^{\prime}$ is the vector of regression mean parameters. At this time we also suppose that the conditional variance $V\left(y_{t} \mid \psi_{t-1}\right)$, depends on the past information through the model $h_{t}=$ $h\left(\alpha_{0}+\alpha_{1} \epsilon_{t-1}^{2}+\ldots+\alpha_{p} \epsilon_{t-p}^{2}\right)$ where $\alpha=\left(\alpha_{0}, \alpha_{1}, \ldots, \alpha_{p}\right)^{\prime}$ is the vector of conditional variance parameters, $\epsilon_{t}=y_{t-1}-x_{t}^{\prime} \beta$ and $h$ is a real monotone function that takes into account the positivity of the variance.

Thus, the stochastic process $\left\{y_{t}\right\}_{t \in I}$ follows a p-order linear ARCH model or $\mathrm{ARCH}(\mathrm{p})$ (Engle 1983) that can be rewritten by:

$$
\begin{gathered}
y_{t} \mid \psi_{t-1} \sim N\left(\mu_{t}, h_{t}\right) \\
\mu_{t}=x_{t}^{\prime} \beta \\
h_{t}=z_{t}^{\prime} w=\alpha_{0}+\alpha_{1} \epsilon_{t-1}^{2}+\ldots+\alpha_{p} \epsilon_{t-p}^{2} \\
\epsilon_{t}=y_{t-1}-x_{t}^{\prime} \beta
\end{gathered}
$$

where $w^{\prime}=\left(\alpha_{0}, \alpha_{1}, \ldots, \alpha_{p}\right)$ and $z_{t}^{\prime}=\left(1, \epsilon_{t-1}^{2}, \epsilon_{t-2}^{2}, \ldots, \epsilon_{t-p}^{2}\right)$.

One way to detect the presence of an ARCH structure, is to apply LjungBox statistics to the results of the correlograms of the residuals from the mean 
model and from the squared residuals (Tsay 2002). Another way is to use a Lagrange multipliers LM test, proposed by Engle (1982) and Bollerslev (1986) in which, after expressing $h_{t}$ as $h_{t}=z_{t 1}^{\prime} \omega_{1}$ and $h_{t}=z_{t 1}^{\prime} \omega_{1}+z_{t 2}^{\prime} \omega_{2}$, we will test the null hypothesis $H_{0}: \omega_{2}=0$. By accepting this hypothesis we demonstrate the dimension of the ARCH effect is not more than the dimension of $\omega_{1}$.

\subsection{GARCH Models}

Let $\left\{y_{t}\right\}_{t \in I}$ be a stochastic process, where $I$ is a discrete process, as given in section 2.1. In the $\operatorname{GARCH}(\mathrm{p}, \mathrm{q})$ regression model the mean model is defined by (2), but the conditional variance models also depends on the conditional variances, as shown by the next equation

$$
h_{t}=z_{t}^{\prime} \omega=\alpha_{0}+\sum_{i=1}^{p} \alpha_{i} \epsilon_{t-i}^{2}+\sum_{i=1}^{q} \gamma_{i} h_{t-i}
$$

where $p \geq 0, q>0, \alpha_{i} \geq 0, \gamma_{i} \geq 0, i=1, \ldots, p$. In this equations $\omega^{\prime}=\left(\alpha_{0}, \alpha_{1}, \ldots\right.$, $\left.\alpha_{q}, \gamma_{1}, \ldots, \gamma_{p}\right)$ and $z_{t}=\left(1, \epsilon_{t-1}^{2}, \ldots, \epsilon_{t-q}^{2}, h_{t-1}, \ldots, h_{t-p}\right)$ are the vectors of the variance parameters and the variance explanatory variables, respectively. If $q=0$, we have an $\mathrm{ARCH}(\mathrm{p})$ regression process.

Usually, GARCH models do not fully reflect the nature of the volatility of most financial assets. These models do not take into account asymmetrical behavior typical of the price volatility of financial assets, which is a wellknown leverage effect. In GARCH models, the conditional volatility of the asset prices is affected symmetrically by positive or negative innovations. In addition, the parameters in the volatility model are restricted to non-negativity. Evidences of this effect have been found by Nelson (1991), Glosten et al. (1993) and Engle \& Ng (1993), among many others.

\subsection{EGARCH Models}

Including the asymmetrical effect given by the fluctuations in the volatility of the asset prices, Nelson (1991) proposed the Exponential class GARCH models, or EGARCH(p,q) models. In this class of models, the conditional variance equation is defined in terms of the standard normal variate $z_{t}=\epsilon_{t} / \sigma_{t}$ and the unexpected log-return through the equation,

$$
\log \left(\sigma_{t}^{2}\right)=\alpha_{0}+\sum_{i=1}^{p} \alpha_{i}\left(\left|z_{t-i}\right|+\lambda_{i} z_{t-i}\right)+\sum_{j=1}^{q} \beta_{j} \log \left(\sigma_{t-j}^{2}\right)
$$

where $\theta=\left(\alpha_{1}, \ldots, \alpha_{p}, \beta_{1}, \ldots, \beta_{q}\right)$ is de vector of parameters. In this model, the effect of asymmetry is considered. Bad news can have a large impact on the volatility, and the values of $\lambda_{i}$ would expected to be negative.

Several studies have found that EGARCH models fit financial data very well, much better than the other GARCH models. The advantages of the exponential specification for the variance are given by many others (see, for example, Taylor 1994, Heynen et al. 1994, Lumsdaine 1995).

Inferences of interest for the ARCH, GARCH and EGARCH models usually are obtained using standard maximum likelihood methods. 


\section{A General EGARCH Model: a Bayesian approach}

Similar to Engle (1982), the $\left\{y_{t}\right\}_{t \in I}$ process follows the autoregressive conditional heteroscedastic model if,

$$
y_{t} \mid \psi_{t-1} \sim N\left(\mu_{t}, h_{t}\right)
$$

$$
\begin{aligned}
& \mu_{t}=x_{t}^{\prime} \beta \\
& h_{t}=\exp \left(\alpha_{0}+\alpha_{1} z_{1, t}+\ldots+\alpha_{p} z_{p, t}\right)
\end{aligned}
$$

where, for example, $z_{i, t}=\epsilon_{t-i}, z_{i, t}=\epsilon_{t-i}^{2}, z_{i, t}=\frac{\epsilon_{t-i}}{h_{t-i}}, z_{i, t}=h_{t-i}$, and $\epsilon_{t}=y_{t}-x_{t}^{\prime} \beta$. The explanatory variables of the volatility model can also include terms of some other financial time series. As in section 2.1, in this model, $\beta$ is the vector of mean parameters, $x_{t}$ is the vector of the mean explanatory variables and $\alpha^{\prime}=\left(\alpha_{0}, \alpha_{1}, \ldots, \alpha_{p}\right)$ is the vector of volatility parameters.

Assuming a general EGARCH model, we could have difficulties in obtaining maximum likelihood estimates for the parameters of the model. A suitable alternative is to use Bayesian methods.

In order to estimate the parameters under the Bayesian methodology, we need a prior distribution for the parameters assessing the information about what we would anticipate as the relative frequency from a very large number of observations. For simplicity, we assign the prior distribution $p(\beta, \alpha) \sim$ $N\left(\theta_{0}, \Sigma_{0}\right)$, where $\theta_{0}^{\prime}=(b, g)$ and $\Sigma_{0}$ is a $(q+p) \times(q+p)$ variance covariance matrix for $\theta=(\beta, \alpha)$. Then, with the likelihood $L(\beta, \alpha)$ given by some distribution that belongs to the two parameter exponential family, and using Bayes theorem, we find the posterior distribution $\pi(\beta, \gamma) \sim L(\beta, \alpha) p(\beta, \alpha)$. Given that $\pi(\beta, \alpha)$ is analytically intractable and not easily generated, we propose sampling $(\beta, \alpha)^{\prime}$ in an iterative process; i.e. sampling $\beta$ from a q-dimensional random walk and $\alpha$ from $\pi(\alpha \mid \beta)$ distribution.

Consequently, if $\beta^{(c)}$ and $\alpha^{(c)}$ are the current values of $\beta$ and $\alpha$, the new values of $\beta=\left(\beta_{0}, \beta_{1}, \beta_{2}, \ldots, \beta_{q}\right)^{\prime}$ are proposed as a multivariate random walk. New values of $\alpha$ are obtained as it is proposed by Cepeda \& Gamerman (2001) from

$$
q_{2}(\alpha \mid \hat{\alpha}, \hat{\beta})=N\left(g^{*}, G^{*}\right)
$$

where $g^{*}=G^{*}\left(G^{-1} g+\frac{1}{2} Z^{\prime} \tilde{Y}\right), G^{*}=\left(G^{-1}+\frac{1}{2} Z^{\prime} Z\right)^{-1}, b$ and $G$ are given by the conditional prior distribution $\gamma \mid \beta \sim N(g, G)$, and $Z$ is the matrix with $\mathrm{t}$-th row equal to $\left(1, z_{1, t}, \ldots, z_{p, t}\right)$ and $\tilde{Y}$ is the $\mathrm{n}$-dimensional vector with the $\mathrm{i}$-th component equal to $\tilde{y}_{i}=\frac{y_{i}-\mu_{i}}{\sigma_{i}}-1$. This proposal is obtained from Fisher scoring. We also could obtain the working variables by Taylor approximation. In this case the working variable is $\tilde{y}_{i}=\frac{y_{i}-\mu_{i}}{\sigma_{i}}$.

\section{Stochastic Volatility Models}

Given the so-called log-returns time series $\left\{y_{t}\right\}_{t \in I}$ this section is devoted to the analysis of volatility models, stated as follows: 


$$
y_{t}=\sigma_{t} \epsilon_{t}, t=1,2, \ldots, n
$$

where $\epsilon_{t}$ is a sequence of i.i.d. random variables with normal distribution $N\left(0, \sigma_{\epsilon}^{2}\right)$. In this model, the volatility of the time series $y_{t}$ is given by the conditional variance $\sigma_{t}^{2}=E\left(y_{t}^{2} \mid H_{t}\right)$, where $H_{t}=\left\{y_{t-1}, y_{t-2} \ldots\right\}$ are the observed values at times $t-1, t-2, \ldots$. Here we assume that the standard deviation is given by the model,

$$
\sigma_{t}=\exp \left\{\frac{h_{t}}{2}\right\}
$$

where $h_{t}$ is a latent variable defined by the autoregressive model

$$
h_{t}=\mu+\phi\left(h_{t-1}-\mu\right)+\eta_{t} \quad t=2,3, \ldots
$$

and we assume that $h_{1}$ is a random variable with known distribution $P_{1}\left(h_{1}\right)$ and $\eta_{t}$ is a sequence of i.i.d. random variables with normal distribution $N(0$, $\left.\sigma_{\eta}^{2}\right)$. If $|\phi|<1$ the mean and the non-conditional variance of $h_{t}$ are $E\left(h_{t}\right)=\mu$ and $\operatorname{Var}\left(h_{t}\right)=\frac{\sigma_{\eta}^{2}}{\left(1+\phi \rho_{1}\right)}$, where $\rho_{1}$ is the coefficient of autocorrelation between $h_{t}$ and $h_{t-1}$ (see for example, Taylor 1994).

With the assumptions (5), (6) and (7), we have

$$
\begin{gathered}
y_{t} \sim N\left(0, \sigma_{\varepsilon}^{2} \exp \left\{h_{t}\right\}\right) \\
h_{1} \sim N\left(\mu, \sigma_{\eta}^{2}\right) \\
h_{t} \mid h_{t-1} \sim N\left(\mu+\phi\left(h_{t-1}-\mu\right), \sigma_{\eta}^{2}\right)
\end{gathered}
$$

for $t=2,3, \ldots n$.

A generalization of the stochastic volatility model, given by (5) and (6), can be obtained by defining a model through (11) for the latent variable defined by

$$
h_{t}=\mu+\sum_{j=1}^{p} \phi_{j}\left(h_{t-j}-\mu\right)+\eta_{t}
$$

for $t=p+1, \ldots, n$, with roots of polynomial $\phi(B)=1-\sum_{j=1}^{p} \phi_{j} B^{j}$ outside the unit circle $\left(B\right.$ is the retarded operator defined by $B^{q} h_{t}=h_{t-q}$ ). The model defined by (5) and (6) and (11) is called Generalized Stochastically Volatility model (GSV).

In this case,

$$
h_{t} \mid h_{t-1}, \ldots, h_{t-p} \sim N\left(\mu+\sum_{j=1}^{p} \phi_{j}\left(h_{t-j}-\mu\right), \sigma_{\eta}^{2}\right)
$$

for $t=2,3, \ldots, n$.

The likelihood function of the GSV, taking into account (5) and (6) is given by the following: 


$$
L=\prod_{t=1}^{n} p\left(y_{t} \mid h_{t}\right)
$$

From (8), we obtain:

$$
L=\prod_{t=1}^{n} \frac{1}{\sqrt{2 \pi \sigma_{\epsilon}^{2} \exp \left\{h_{t}\right\}}} \exp \left\{-\frac{y_{t}^{2}}{2 \sigma_{\epsilon}^{2} \exp \left\{h_{t}\right\}}\right\}
$$

With this new generalized stochastic Volatility Model, we can obtain better inferences for the volatilities of financial time series.

\section{Bayesian analysis of GSV model}

For a Bayesian analysis of the Stochastic volatility model defined by (5) and (6), with latent variables defined by (11), we assume the following prior distributions for $\mu, \Phi=\left(\phi_{1}, \phi_{2}, \ldots, \phi_{p}\right)^{\prime}, \sigma_{\epsilon}^{2}$ and $\sigma_{\eta}^{2}$.

$$
\begin{gathered}
\phi_{j} \sim B\left(a_{j}, b_{j}\right), j=1, \ldots, p \\
\sigma_{\epsilon}^{2} \sim I G\left(c_{1}, d_{1}\right) \\
\sigma_{\eta}^{2} \sim I G\left(c_{2}, d_{2}\right) \\
\mu \sim N\left(0, e^{2}\right)
\end{gathered}
$$

where $B\left(a_{j}, b_{j}\right)$ denotes a beta distribution with mean $\frac{a_{j}}{\left(a_{j}+b_{j}\right)}$ and variance $\frac{a_{j} b_{j}}{\left[\left(a_{j}+b_{j}\right)^{2}\left(a_{j}+b_{j}+1\right)\right]} ; I G(c, d)$ denotes the inverse gamma distribution with mean $\frac{d}{(c-1)}$ and variance $\frac{d^{2}}{\left[(c-1)^{2}(c-2)\right]}, c>2$, and $N\left(\mu, \sigma^{2}\right)$ denotes the normal distribution with mean $\mu$ and variance $\sigma^{2}$. We assume that the hyperparameters $\left(a_{j}, b_{j}\right), j=1, \ldots, p,\left(c_{i}, d_{i}\right), i=1,2$, and $e^{2}$ are known.

If $h_{l}=0$, for $l=0,-1, \ldots,-p+1$ in (12), the conditional distribution of $h_{t}$ given $h_{t-1}^{(p)}=\left\{h_{t-1}, \ldots h_{t-p}\right\}$, for $t=1, \ldots, n$, is given by

$$
p\left(h_{t} \mid h_{t-1}^{(p)}\right)=\frac{1}{\sqrt{2 \pi \sigma_{\eta}^{2}}} \exp \left\{-\frac{1}{2 \sigma_{\eta}^{2}}\left[h_{t}-\mu-\sum_{j=1}^{p} \phi_{j}\left(h_{t-j}-\mu\right)\right]^{2}\right\}
$$

Thus, if $\Phi=\left(\phi_{1} \ldots, \phi_{p}\right)^{\prime}, \theta=\left(\mu, \Phi, \sigma_{\epsilon}^{2}, \sigma_{\eta}^{2}\right), h=\left(h_{1}, \ldots, h_{n}\right)^{\prime}$ and if $\mu, \Phi, \sigma_{\epsilon}^{2}, \sigma_{\eta}^{2}$ have independent prior distributions, the posterior density function for $\varphi=$ $(\theta, h)$ is given by 


$$
\begin{aligned}
\pi(\varphi \mid y)= & \prod_{t=1}^{n} \frac{1}{\sqrt{2 \pi \sigma_{\epsilon}^{2} \exp \left\{h_{t}\right\}}} \exp \left\{-\frac{y_{t}^{2}}{2 \pi \sigma_{\epsilon}^{2} \exp \left\{h_{t}\right\}}\right\} \\
& \times \frac{1}{\sqrt{2 \pi \sigma_{\epsilon}^{2}}} \exp \left\{-\frac{1}{2 \sigma_{\eta}^{2}}\left[h_{t}-\mu-\sum_{j=1}^{p} \phi_{j}\left(h_{t-j}-\mu\right)\right]\right\}^{2} \pi(\theta)
\end{aligned}
$$

where $y=\left(y_{1}, \ldots, y_{n}\right)^{\prime}$ and $\pi(\theta)$ is given by

$$
\begin{aligned}
\pi(\theta) \propto & \left\{\prod_{j=1}^{p} \phi_{j}^{a_{j-1}}\left(1-\phi_{j}\right)^{b_{j-1}}\right\}\left(\sigma_{\eta}^{2}\right)^{-\frac{\left(c_{1}+1\right)}{2}} \exp \left\{-\frac{d_{1}}{\sigma_{\eta}^{2}}\right\} \\
& \times\left(\sigma_{\epsilon}^{2}\right)^{-\frac{\left(c_{2}+1\right)}{2}} \exp \left\{-\frac{d_{2}}{\sigma_{\eta}^{2}}\right\} \exp \left\{-\frac{\mu^{2}}{2 e^{2}}\right\}
\end{aligned}
$$

Thus, the posterior density can be rewritten as

$$
\begin{aligned}
\pi(\varphi \mid y) \propto & \left(\sigma_{\epsilon}^{2}\right)^{-\frac{n}{2}} \exp \left\{-\frac{1}{2} \sum_{t=1}^{n} h_{t}-\frac{1}{2 \sigma_{\epsilon}^{2}} \sum_{t=1}^{n} y_{t}^{2} \exp \left\{-h_{t}\right\}\right\} \\
& \times\left(\sigma_{\eta}^{2}\right)^{-\frac{n}{2}} \exp \left\{-\frac{1}{2 \sigma_{\eta}^{2}} \sum_{t=1}^{n}\left(h_{t}-\mu-\sum_{j=1}^{p} \phi_{j}\left(h_{t-j}-\mu\right)\right)^{2}\right\} \pi(\theta)
\end{aligned}
$$

Finally, if $\Phi=\left(\phi_{1} \ldots, \phi_{p}\right)^{\prime}, y=\left(y_{1}^{2}, \ldots, y_{n}^{2}\right), h=\left(h_{i}, \ldots, h_{n}\right)^{\prime}, E(h)=\left(e^{-h_{i}}, \ldots, e^{-h_{N}}\right)^{\prime}$ and the matrix $X$ given by:

$$
X=\left(\begin{array}{cccc}
h_{0} & h_{-1} & \ldots & h_{-p+1} \\
h_{1} & h_{0} & \ldots & h_{-p+2} \\
\vdots & \vdots & \ldots & \vdots \\
h_{p} & h_{p-1} & \ldots & h_{1} \\
h_{p+1} & h_{p} & \ldots & h_{2} \\
\vdots & \vdots & \ldots & \vdots \\
h_{n-1} & h_{n-2} & \ldots & h_{n-p}
\end{array}\right)_{n \times p},
$$

the posterior density (20) can be written as

$$
\begin{aligned}
\pi(\varphi \mid y) \propto & \left(\sigma_{\epsilon}^{2}\right)^{-\frac{n}{2}} \exp \left\{-\frac{1}{2} 1^{\prime} h-\frac{1}{2 \sigma_{\epsilon}^{2}} y^{\prime} E(h)\right\} \\
& \times\left(\sigma_{\eta}^{2}\right)^{-\frac{n}{2}} \exp \left\{-\frac{1}{2 \sigma_{\eta}^{2}}\left(h-\mu-X^{\prime} \Phi\right)^{\prime}\left(h-\mu-X^{\prime} \Phi\right)\right\} \pi(\theta)
\end{aligned}
$$

where $1=(1, \ldots, 1)^{\prime}$ is the vector $n \times 1$ of $1^{\prime} s$ and $\mu$ is de vector of $n \times 1$ of $\mu^{\prime} s$. 
Sampling from the joint posterior distribution of $\varphi(\theta, h)$, we use MCMC methods with Gibbs Sampling algorithm (Gelfand \& Smith 1990) or MetropolisHastings (Smith \& Roberts 1993). These samples are generated from the conditional distributions $\phi\left(\theta_{j} \mid \theta_{(j)}, y\right)$, where $\theta_{(j)}$ denotes the vector of all components of $\theta$, except the $j-$ th component.

\section{An Application}

As an application, we analyze the time series of prices and log-returns of share prices of the Gillette Company, taken daily at the closing of the market, from January, 1999 to May, 2003. The results were obtained using the statistical program Eviews 5.0, for ARCH, GARCH and EGARCH models under the classical inference approach, and WinBugs software (Spiegelhalter et al. 1999) under Bayesian inference approach. Moreover, the selection procedure was based on the AIC (Akaike Information Criterion) and BIC (Bayes Information Criterion). In all cases, the $\mathrm{Q}$ statistics of Ljung-Box do not reject the nullhypothesis ${ }^{1}$

BIC (Bayes Information Criterion) is a model discrimination criterion introduced by Schwarz (1978) and modified by Carlin \& Louis (2000) to be applied assuming the posterior density for the parameters of the fitted model. This criterion weights between the maximized likelihood function and the number of parameters of the model. The best model is the one with larger value of BIC.

\subsection{Models for shares' prices}

In order to illustrate the results provided by the models discussed in this paper we took Gillette's stock prices time series on a daily basis at the closing market index lasting from January, 1999 to May, 2003. Figure 1 shows the behavior of this time series and what strikes us most is a general descending feature of these prices. This behavior is surely explained by the microeconomic aspects of this Company, once this used to be a typical period of stock market boom.

Observing the pattern showed in Figure 1, it is quite precise to state that there are four price cycles lasting one year each. Probably, as a stylized fact, this finding is linked to the product's life cycle released to the market on an annual basis. Gillette is an internationally well-known brand of men's safety razors, amongst other personal care products and it used to be a leading global supplier of these products until it merged with Procter \& Gamble in 2005. It appears to be the Company's strategies on releasing new products followed a life cycle as expected, but these strategies were also accompanied by a steady decrease in the Company's market value.

After taking a closer look at Gillette's history since the 70's, we can see that this Company went through takeover attempts more than once and faced judicial dispute claims for its products prior to merging with P\&G in 2005.

From the macroeconomic point of view Gillette's stock prices diverged from the expected behavior of stock market prices in this time period. Stock

\footnotetext{
${ }^{1} \mathrm{H}$ : there is no serial correlation for residuals, as for square residuals., at a $1 \%$ significance level.
} 
prices data for Gillette covers roughly the second term of Clinton's administration in USA, a period of economic and financial boom. In recent times this period demonstrated one of the most economic and financial growing virtuous cycle, contrasting with the poor performance of Gillette's stock prices.

Besides these incidents in corporate management, Gillette also faced the fact that it used to be a traditional Company clearly affected by issues such as technology decline, more efficient competitors, amongst others. Definitely, Gillette did not introduce itself into the technological development stream that characterized the time period analyzed. After merging with Procter \& Gamble in 2005, Gillette faced its dissolution and was finally incorporated as a division of $P \& G$ in 2007.

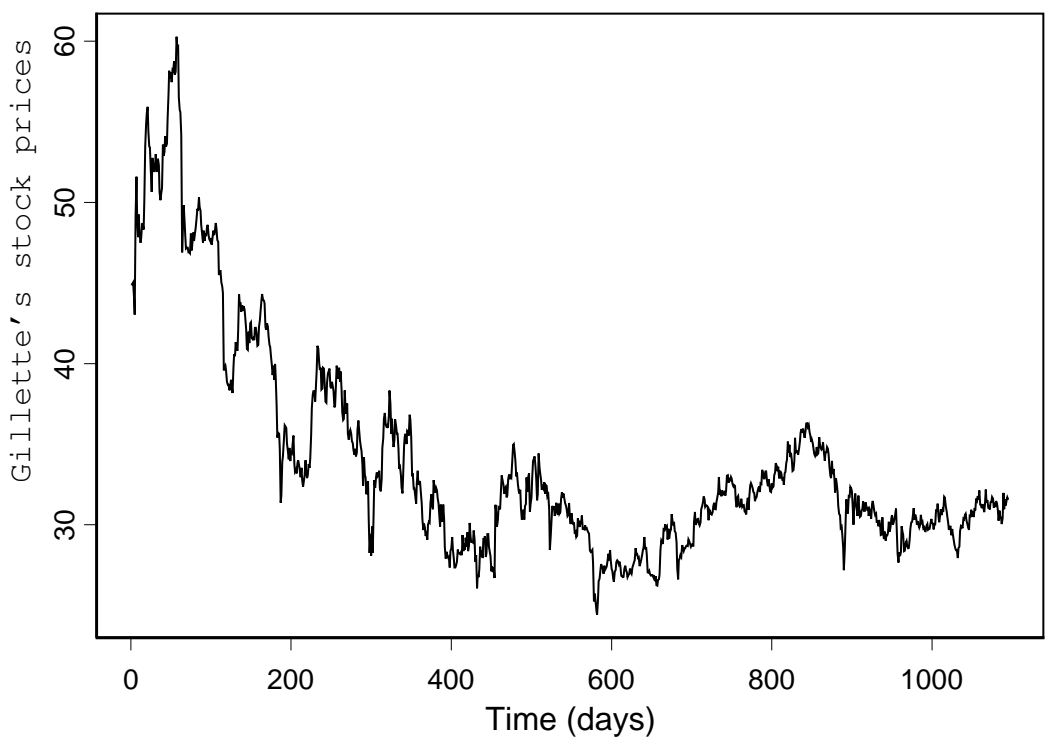

Figure 1: Time series of the prices.

\section{ARCH Models}

Among different ARCH models, the $\mathrm{ARCH}(4)$ model has the smallest BIC value. The maximum likelihood estimates (MLE) of the mean and variance parameters, and their standard deviations, are given in Table 1.

Table 1: Maximum likelihood parameter estimates of $\mathrm{ARCH}(4)$ model.

\begin{tabular}{crcccccc}
\hline Parameter & $\beta_{0}$ & $\beta_{1}$ & $\alpha_{0}$ & $\alpha_{1}$ & $\alpha_{2}$ & $\alpha_{3}$ & $\alpha_{4}$ \\
\hline Mean & -0.1350 & 1.005 & 0.2195 & 0.4652 & 0.0826 & 0.2009 & 0.0892 \\
s. d. & 0.0610 & 0.001 & 0.0199 & 0.0398 & 0.0277 & 0.0306 & 0.0179 \\
\hline
\end{tabular}

The $\mathrm{p}$-values associated with the tests $H: \theta=0$ versus $\theta \neq 0$, where $\theta$ represents the parameters $\alpha_{0}, \alpha_{1}, \alpha_{2}, \alpha_{3}, \alpha_{4}$ or $\beta_{0}$ and $\beta_{1}$, are all smaller than 
0.004 , except the $\mathrm{p}$-value associated with $\beta_{0}$, that is given by 0.027 . The AIC and BIC values are given by 2.2201 and 2.2524 , respectively.

\section{GARCH Models}

Among different GARCH models, the $\operatorname{GARCH}(1,2)$ model has the smallest BIC value. The MLE estimates of the mean and variance parameters, and their standard deviations are given in Table 2.

Table 2: Maximum likelihood parameter estimates of $\operatorname{GARCH}(1,2)$ model.

\begin{tabular}{ccccccc}
\hline Parameter & $\beta_{0}$ & $\beta_{1}$ & $\alpha_{0}$ & $\alpha_{1}$ & $\alpha_{2}$ & $\gamma_{1}$ \\
\hline Mean & 0.4566 & 0.9857 & 0.2195 & 0.4656 & 0.0826 & 0.2009 \\
s. d. & 0.1583 & 0.0044 & 0.0199 & 0.0398 & 0.0277 & 0.0306 \\
\hline
\end{tabular}

The $\mathrm{p}$-values associated with the test $\mathrm{H}: \theta=0$ versus $\theta \neq 0$, where $\theta$ represents all regression parameters, are all smaller than 0.006. The AIC and BIC values are given by 2.1059 and 2.1336 , respectively.

\section{General EGARCH Models}

In this section, we fit two models to analyze the time series of prices and logreturns of Shares of the Gillette Company, applying the Bayesian methodology given in section 3 . In both models, $y_{t} \mid \psi_{t-1} \sim N\left(\mu_{t}, h_{t}\right)$ with $\mu_{t}=\beta_{0}+\beta_{1} y_{t-1}$, where $\beta^{\prime}=\left(\beta_{0}, \beta_{1}\right)$ is the mean parameter vector.

We assume normal prior distribution, $N\left(0,10^{k}\right)$, for all parameters with large variances, $k=5$ to have approximately non informative priors, considering two different models for the prices.

Model 1. Here we assume that the conditional variance of the stochastic process $Y_{t, t \in T}$ is given by the model

$$
h_{t}=\exp \left(\alpha_{0}+\alpha_{1} \epsilon_{t-1}^{2}+\alpha_{2} \epsilon_{t-2}\right)
$$

where $\alpha^{\prime}=\left(\alpha_{0}, \alpha_{1}, \alpha_{2}\right)$ is the vector of variance parameters and $\epsilon_{t}=y_{t}-x_{t}^{\prime} \beta$. The parameter estimates (posterior means) of this model are given in Table 3. Figure 2 shows the behavior of the chain sample for each parameter, each one of which has a small transient stage, indicating the speed convergence of the algorithm. The chain samples are given for the first 4500 iterations. The other results reported in this section are based on a sample of 4000 draws after a burn-in of 1000 draws.

Table 3: Posterior summaries for the prices model (model 1)

\begin{tabular}{cccccc}
\hline Parameter & $\beta_{0}$ & $\beta_{1}$ & $\alpha_{0}$ & $\alpha_{1}$ & $\alpha_{2}$ \\
\hline Mean & -0.0222 & 1.0008 & -0.7402 & 0.3183 & -0.1768 \\
s.d. & 0.1182 & 0.0035 & 0.0551 & 0.0499 & 0.0462 \\
\hline
\end{tabular}


The histograms of the generated samples for each parameter seem to show that the posterior marginal distribution for all the parameters is approximately normal. Figure 3 shows the histograms for the posterior marginal distributions of the variance parameters.

According to a theoretical result in which the model where the information matrix is not block diagonal, Table 4 shows a large correlation between mean and variance parameters. For this model the AIC and BIC values are given by 2.330 and 2.3228 , respectively.

Table 4: Posterior summaries for the correlation between the parameters (model 1)

\begin{tabular}{crrrcc}
\hline Parameters & \multicolumn{1}{c}{$\beta_{0}$} & $\beta_{1}$ & $\alpha_{0}$ & $\alpha_{1}$ & $\alpha_{2}$ \\
\hline$\beta_{0}$ & 1 & & & & \\
$\beta_{1}$ & -0.976 & 1 & & & \\
$\alpha_{0}$ & 0.121 & -0.156 & 1 & & \\
$\alpha_{1}$ & -0.196 & 0.230 & -0.633 & 1 & \\
$\alpha_{2}$ & 0.156 & -0.141 & 0.178 & -0.234 & 1 \\
\hline
\end{tabular}
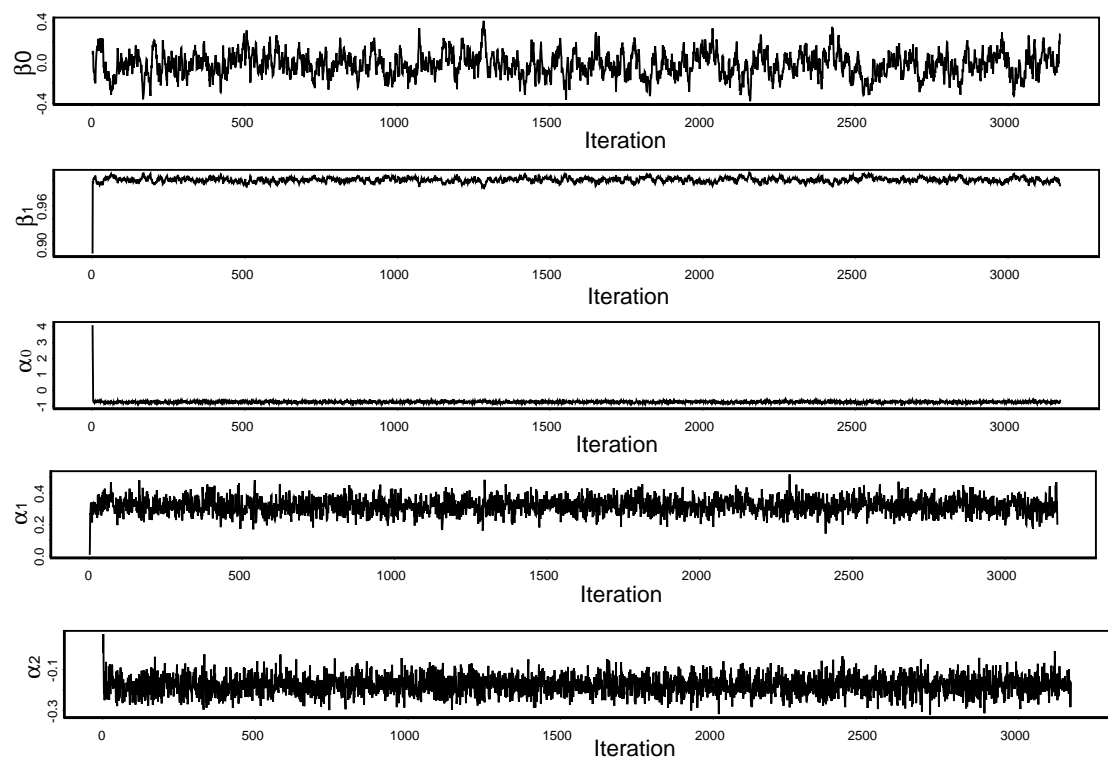

Figure 2: Time series plots for the simulated sample for each parameter

Model 2. Here we assume that the conditional variance of the stochastic process $Y_{t, t \in I}$ is given by the model,

$$
h_{t}=\exp \left(\alpha_{0}+\alpha_{1} \epsilon_{t-1}^{2}+\alpha_{2} \epsilon_{t-2}^{2}\right)
$$

where $\alpha^{\prime}=\left(\alpha_{0}, \alpha_{1}, \alpha_{2}\right)$ is the vector of variance parameters and $\epsilon_{t}=y_{t}-x_{t}^{\prime} \beta$. 

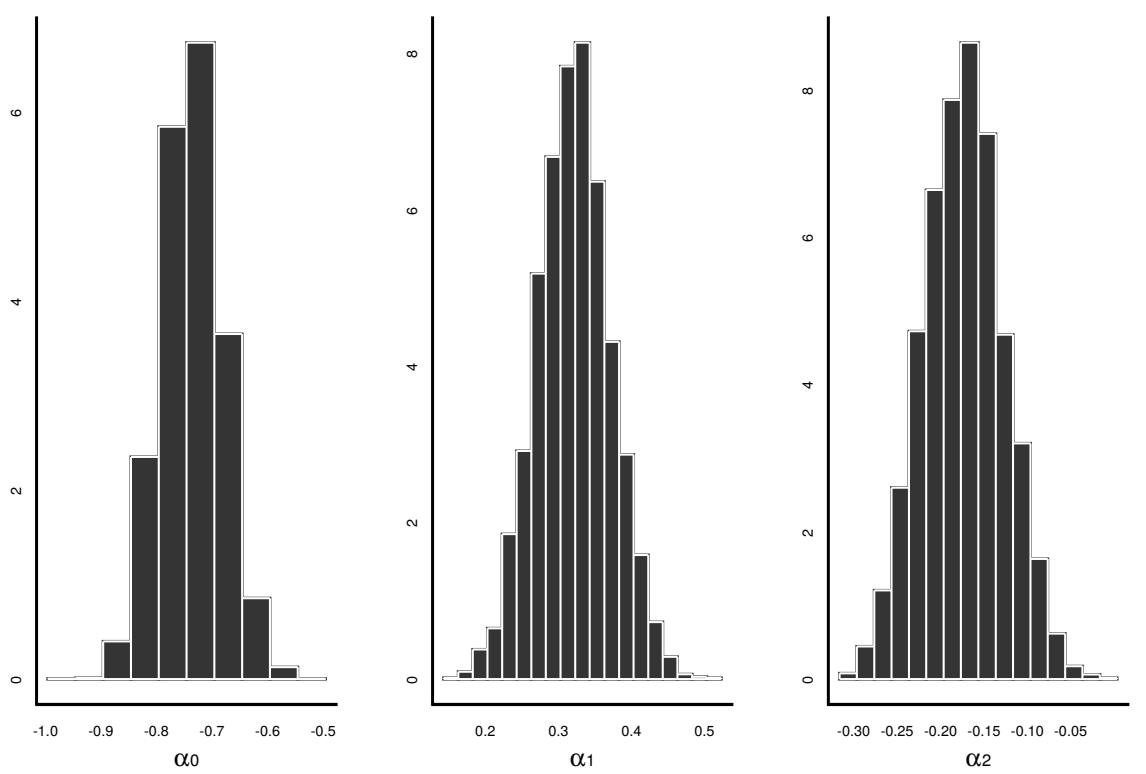

Figure 3: Marginal posterior distribution for the variance parameters

The posterior samples were recorded every 10th sample, after a burn-in period of 1,000 Gibbs samples, to have approximately uncorrelated samples. The posterior summaries for this model are given in Table 5.

Table 5: Posterior summaries for the prices model (model 2)

\begin{tabular}{cccrcc}
\hline Parameter & $\beta_{0}$ & $\beta_{1}$ & $\alpha_{0}$ & $\alpha_{1}$ & $\alpha_{2}$ \\
\hline Mean & 0.0473 & 0.9989 & -0.7217 & 0.2714 & 0.0395 \\
s. d. & 0.1327 & 0.0040 & 0.0553 & 0.0533 & 0.0229 \\
\hline
\end{tabular}

In this case, each one of the chain shows small transient stage, indicating the speed convergence of the algorithm. The histograms for the generated samples for the parameters also show that the posterior marginal distributions for all the parameters are approximately normal, and the correlation between posterior parameter sampling shows that $0.11<\operatorname{corr}\left(\beta_{i}, \alpha_{j}\right)<0.31$, that is, a small value, but all significatively different from zero. The AIC and BIC values for this model are given by 2.3198 and 2.3234, respectively.

From the obtained results for all assumed models, we observed that model 1 is a best model fit for time series of prices for Gillette, since the BIC value associated with this model is the smallest when compared to the other models.

\subsection{Models for the log-return Gillette series}

Let $P_{t}, t=1.2, \ldots$, be the price of an asset over time $t$. Assuming that the asset does not pay dividends, its tenure by a time period, from $t-1$ to $t$, generates a log-return sample, defined by

$$
R_{t}=\frac{P_{t}}{P_{t-1}}-1=\frac{P_{t}-P_{t-1}}{P_{t-1}}
$$


In this period of time the log-returns, $r_{t}$, are defined by,

$$
r_{t}=\ln \left(R_{t}+1\right)=\ln \left(P_{t}\right)-\ln \left(P_{t-1}\right)
$$

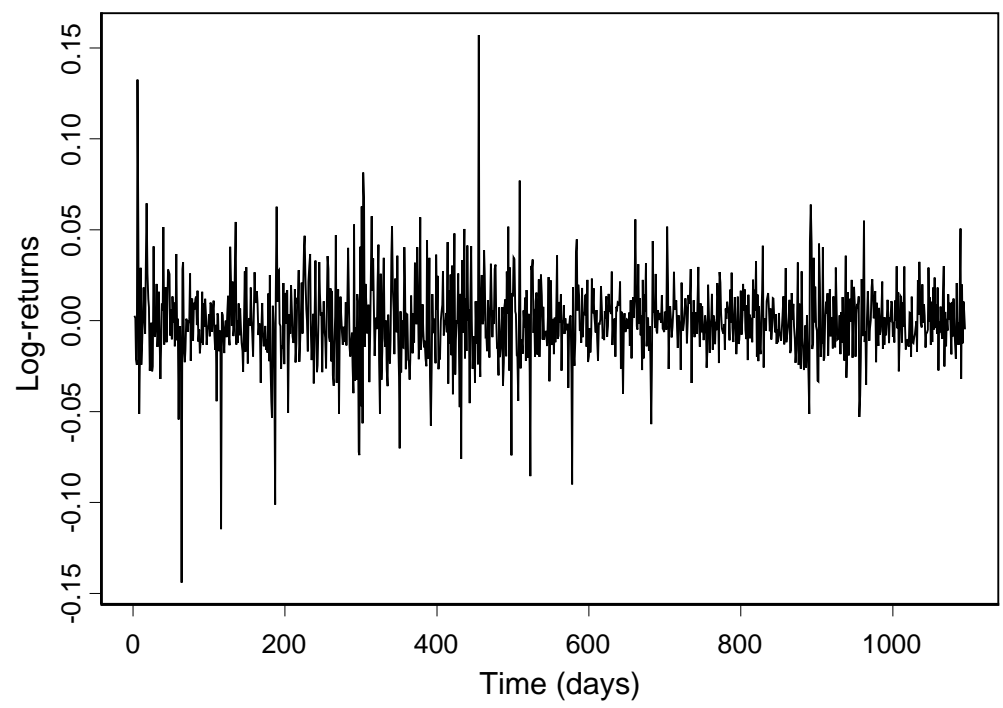

Figure 4: Time series of log-returns of the Gillette Company stock

Figure 4 represents the log-return behavior of Gillette's stock prices for the same period. Although the loss of value faced by the company is a feature inferred by price behavior, changing volatility pattern is a stylized fact deduced from this picture. It is evident there are two striking behaviors for returns volatility, a broader one from 1999 to the beginning of 2002 and a narrower one from 2002 on. This picture describes a situation that shows more volatility during the time Gillette was losing more value, an expected fact because stockholders are more sensitive when their losses are bigger. Shareholders who detain broader slices of the company's control are expected to remain until the complete company's selling, otherwise their losses can be even bigger. Probably this explains the company's diminishing stock prices volatility after 2002.

Assuming Gillette stockholders are experiencing loss of value of their shares, the return volatility picture shows more volatility between 1999 and 2002, which coincides with the period Gillette was facing more abrupt problems in its management. After 2002 to the end of the period this time series was taken, Gillette experienced less volatility, probably reflecting the conclusion of the merger with $P \& G$ was concluded. Certainly, the mean value estimated foreturns and volatility resembles the returns behavior change after 2002. But obviously volatility is an issue for pricing Gillette's main asset: its share price. 


\section{Models for the log-returns series}

For modeling log returns of asset prices models of the classes GARCH and EGARCH are mostly used and estimated, once the residuals and the square of residuals satisfy the autocorrelation principle. The selected estimated model for the series of log-returns of share' prices of Gillette is the $\operatorname{EGARCH}(2,1)$, with:

$$
\begin{aligned}
\hat{\mu}_{t}= & -0.0007 \\
\ln \left(\hat{h}_{t}\right)= & -0.1014+0.9910 \ln \left(\hat{h}_{t-1}\right)+0.338\left|z_{t-1}\right|-0.1467 z_{t-1} \\
& -0.2973\left|z_{t-2}\right|+0.0724 z_{t-2}
\end{aligned}
$$

where $\hat{\mu}_{t}$ is the MLE estimate of the mean, $\hat{h}_{t}$ is the MLE estimate of the variance and $z_{t}=\frac{\epsilon_{t}}{\sqrt{\hat{h}_{t}}}$ is the standardized residual deviation. The $\mathrm{p}$-values of the tests for all parameters are all smaller than 0.001, except the p-value associated with the parameter $\gamma_{2}$, which is given by 0.045 . For this model the AIC and BIC values are -4.9383 and -4.9062 , respectively.

\section{General Exponential GARCH Model}

In this section we consider the $\left\{r_{t}\right\}_{t \in I}$ process, where $r_{t}$ is the log-return at time $t$ centered in zero. In this case we consider two very simple models. The first one is given by the equation

$$
h_{t}=\exp \left(\alpha_{0}+\alpha_{1}\left|r_{t-1}\right|+\alpha_{2} r_{t-2}\right)
$$

has Bayesian parameter estimates (and standard deviations) given in Table 6. For this model, the $A I C$ and $B I C$ values are equal to -3.8590 and -3.8454 , respectively.

Table 6: Posterior summaries for General Exponential model

\begin{tabular}{cccr}
\hline Parameter & $\alpha_{0}$ & $\alpha_{1}$ & \multicolumn{1}{c}{$\alpha_{2}$} \\
\hline Mean & -8.098 & 25.970 & -4.345 \\
s. d. & 0.0631 & 2.878 & 1.808 \\
\hline
\end{tabular}

The second one given by the equation

$$
h_{t}=\exp \left(\alpha_{0}+\alpha_{1}\left|r_{t-1}\right|^{\frac{1}{2}}+\alpha_{2} r_{t-1}+\alpha_{3} r_{t-2}\right)
$$

has Bayesian parameter estimates (and standard deviations) given in Table 7. For this model, the $A I C$ value are equal to -3.8767 and $B I C$ values are equal to -3.8631 . Thus, the last model is better suited by the data using AIC and BIC as the selection criterion, since it has the smallest value.

The simulated MCMC samples were obtained using the same procedure shown in section 3, and normal prior distribution for all parameters with a large variance. 
Table 7: Posterior summaries for General Exponential model

\begin{tabular}{ccccc}
\hline Parameter & \multicolumn{1}{c}{$\alpha_{0}$} & \multicolumn{1}{c}{$\alpha_{1}$} & \multicolumn{1}{c}{$\alpha_{2}$} & $\alpha_{3}$ \\
\hline Mean & -8.483 & 6.937 & -6.2050 & -6.937 \\
s. d. & 0.0972 & 0.7762 & 1.9730 & 0.7762 \\
\hline
\end{tabular}

\section{Stochastic Volatility Model}

Assuming the stochastic volatility model introduced in section 4 by equations 4, 5 and 5, with $\sigma_{\epsilon}=1$, and prior distributions $13,14,15$ and 16 with hyperparameter values $a_{1}=b_{1}=c_{1}=c_{2}=1$ and $e^{2}=100$, we have in Table 8 , the posterior summaries of interest based on a final Gibbs sample of size 1000 and a burn-in-sample of 5000 samples using the software WinBugs.

Table 8: Posterior summaries (SV model)

\begin{tabular}{cccc}
\hline Parameters & $\mu$ & $\phi$ & $\sigma_{\eta}$ \\
\hline Mean & -8.076 & 0.7661 & 3.132 \\
s. d. & 0.09724 & 0.05706 & 0.7626 \\
\hline
\end{tabular}

For this model, the AIC and BIC values are -5.3819 and -5.3682 , respectively. Observe that based on the AIC and BIC values, the SV models with AR(1) structure give a better fit for the log-returns of Gillette, since they have smaller AIC and BIC values than using the general exponential GARCH models. In the same way, based on these criteria, we observe that SV provides a better fit for the log-return time series when compared to the $\operatorname{EGARCH}(2,1)$ model.

\section{Concluding Remarks}

Different volatility models have been introduced in the literature to analyze financial time series. Using Bayesian methods is a suitable alternative to analyzing the volatility of financial time series, since the complexity of the likelihood function can be a problem for obtaining maximum likelihood estimates for the parameters of the proposed models, especially when we assume general exponential GARCH models and SV models.

For the example of financial time series included in this paper, we observe that SV models give better fit for the data, especially for the log-return series.

Further studies should be carried on for other applications mainly in the special cases of multivariate time series.

It is important to point out that General Exponential GARCH models or SV models usually provide improvements in the fit of time series data when compared to the usual ARCH or GARCH models as we can see by the results achieved in this paper.

A set of four different models are estimated in this paper for the stock prices of Gillette and their estimates are presented in section 6. From the usual estimates of ARCH, GARCH and EGARCH models, two stylized facts 
are confirmed: volatility is an issue to be concerned with and its incidence over the stock prices is skewed. Excess volatility is inferred by its parameters significance and distortion is demonstrated by the signal changes in EGARCH estimates.

Concerning log-returns estimates, Bayesian estimates are a better fit. Among three estimated models, a Bayesian stochastic volatility model representation turns out to be the best one, a conclusion that is based on AIC and BIC criteria. Bayesian stochastic volatility model estimates outperform others because introducing prior distributions to each parameter is a more flexible inference procedure. Moreover, the possibility of introducing an order one autoregressive structure to represent the noise better fits Gillette's stock returns.

The obtained results allow us to conclude that an order one autoregressive process is significant in determining returns of this Company in this period of time; its return is almost zero on average and total volatility is expressive, a statement that is confirmed by the estimate of $\sigma_{\eta}$. Clearly these results resembles the main features of the log-returns contained in Figure 4 and validates the hypotheses of significance of null expected average returns; autoregressive behavior for volatility of financial time series and the excess volatility are presented in these time series.

All the estimates provided by ARCH, GARCH and EGARCH class models confirm the expected excess volatilityshowed by financial time series. Moreover, the long lasting memory volatility hypothesis is accepted in both cases; i.e. for the error term and for the entire volatility structure. In particular, EGARCH models reflect the leverage effect that weighs more bad news than good news' likelihood. Changing estimates signals and their magnitudes strengthen these results.

The magnitudes of $\beta_{1}$ estimates in all models reinforce the idea of time series integration, a common feature in financial asset prices. However, expressing the model in returns the problem is overcomed, as expected. Possibly, this finding is a matter for demanding the introduction of a autoregressive structure into the latent variable, what is successfully done when estimating SV Bayesian Models.

Concerning SV Bayesian models, it is clear its results strictly reproduce the main features presented by finamcial asset prices time series. Volatility is an issue, since it is expressive and significant by the estimates presented in table 6 . With a coefficient magnitude of 3.132 for $\sigma_{n}$ the investor can expect a deviation of approximately $4.78 \%$ from the mean return, which is significant for a US stock market perspective.

Autoregressive coefficient is also significant and its estimated value reflects the existence of a memory structure on determining returns volatility. Roughly, $75 \%$ of a volatility generated in a period of time is transmitted to the following period, which is a despicable mark. Finally, the estimated mean returns resembles the one exhibited in figure 6; i.e. though the estimated coefficient is negative and equal to -8.076 , we remember this is a result for returns in logarithm, so the real percentage return is close to zero.

\section{Bibliography}

Black, F. \& Scholes, M. (1973), 'The pricing of options and corporate liabilities', The journal of political economy pp. 637-654. 
Bollerslev, T. (1986), 'Generalized autoregressive conditional heteroskedasticity', Journal of Econometrics 31, 307-327.

Carlin, B. \& Louis, T. (2000), Bayes and Empirical Bayes methods for date analysis, Chapman and Hall/ CRC Press, -.

Cepeda, E. \& Gamerman, D. (2001), 'Bayesian modelling of variance heterogeneity in normal regression models', Brazilian Journal of Probability and Statistics 14, 207-221.

Engle, R. (1982), 'Autoregressive conditional heteroscedasticity with estimates of the variance of uk inflation', Econ. 50, 987-1007.

Engle, R. (1983), 'Estimates of the variance of us inflation based upon the arch model', Journal of Money, Credit, and Banking 15(286-301).

Engle, R. F. \& Ng, V. (1993), 'Measuring and testing the impact of news on robert engle volatility', Journal of Finance 48(5), 1749-1778.

Gelfand, A. E. \& Smith, A. F. M. (1990), 'Sampling-based approaches to calculating marginal densities', Journal of the American Statistical Association 85(410), 398-409.

Ghysels, E., Harvey, A. C. \& Renault, E. (1996), 'Stochastic volatility', Statistical methods in finance. Handbook of Statist pp. 119-191.

Glosten, L., Jannanthan, R. \& Runkle, D. (1993), 'Relationship between the expected value and the volatility of the nominal excess of return on stocks', Journal of Finance 48, 1779-1802.

Heynen, R., A., K. \& T., V. (1994), 'Analysis of the term structure of implied volatilities', Journal of Financial and Quantitative Analysis 29, 31-56.

Hull, J. \& White, A. (1987), 'The pricing of options on assets with stochastic volatility', The Journal of Finance 42(2), 281-300.

Kim, S., Shephard, N. \& Chib, S. (1998), 'Stochastic volatility: likelihood inference and comparison with arch models', The Review of Economic Studies 65(3), 361-393.

Lumsdaine, R. (1995), 'Finite-sample properties of the maximum-likelihood estimator in $\operatorname{garch}(1,1)$ and igarch $(1,1)$ models a monte carlo investigation', Journal of Business and Economic Statistics 13, 1-10.

Meyer, R. \& Yu, J. (2000), 'Bugs for a bayesian analysis of stochastic volatility models', The Econometrics Journal 3(2), 198-215.

Nelson, B. D. (1991), 'Conditional heterocedasticity in asset returns: A new approach', Econometrica 59(2), 347-370.

Schwarz, G. (1978), 'Estimation of the dimension of a model', Annals of Statistics 6, 461-466.

Shephard, N. (1996), 'Statistical aspects of arch and stochastic volatility', Monographs on Statistics and Applied Probability 65, 1-68. 
Smith, A. F. M. \& Roberts, G. O. (1993), 'Bayesian computation via the gibbs sampler and related markov chain monte carlo methods (with discussion)', Journal of the Royal Statistical Society. B 55, 3-23.

Spiegelhalter, D. J., Thomas, A., Best, N. G. \& Lund, D. (1999), WinBUGS User Manual, Cambridge, United Kingdon: MRC Biostatistics Unit.

Taylor, S. (1994), 'Modelling stochastic volatility: a review and comparative study', Mathematical finance 4, 183-204.

Tsay, R. S. (2002), Analysis of financial time series, Wiley, New York. 